\title{
CARACTERÍSTICAS DEL POLICONSUMO SIMULTÁNEO DE DROGAS EN ESTUDIANTES DE PREGRADO DE CARRERAS DE CIENCIAS DE LA SALUD DE UNA UNIVERSIDAD, SANTIAGO - CHILE
}

\author{
Giselle Riquelme Hernández¹, Laura Simich², Carol Strike ${ }^{3}$,Bruna Brands', Norman Girsbrecht ${ }^{5}$, Akwatu \\ Khenti ${ }^{6}$
}

\footnotetext{
${ }^{1}$ MSc en Enfermería. Profesora Asistente Adjunta del Departamento Salud de la Mujer. Escuela de Enfermería, Pontificia Universidad Católica de Chile. Santiago, Chile. E-mail: gfriquel@uc.cl

${ }^{2}$ Ph.D. Researcher. Health Systems Research and Consulting Unit. Centre for Addiction and Mental Health. University of Toronto. Canada. E-mail: laura_simch@camh.net

${ }^{3}$ Ph.D. Researcher. Health Systems Research and Consulting Unit. Centre for Addiction and Mental Health. University of Toronto. Canada. E-mail: carol_strike@camh.net

${ }^{4}$ Ph.D. Researcher. Office of Research and Surveillance, Drug Strategy and Controlled Substances Programme. Health Canada and Public Health and Regulatory Policies. Center for Addiction and Mental Health. University of Toronto. Canada. E-mail: bruna_brands@camh.net

${ }^{5}$ Ph.D. Researcher. Health Systems Research and Consulting Unit. Centre for Addiction and Mental Health. University of Toronto. Canada. E-mail: norman_giebsbrecht@camh.net

${ }^{6}$ MSc. Director of Office of International Health Center for Addiction and Mental Health. Toronto, Canada. E-mail: akwatu_ khenti@camh.net
}

RESUMEN: Estudio transversal cuyo objetivo fue establecer los patrones de policonsumo simultáneo de drogas en estudiantes universitarios de pregrado de carreras de medicina y enfermería, en una universidad de la ciudad de Santiago - Chile. Contó con una muestra de 188 estudiantes. Resultados: 42.3\% de los estudiantes refirieron policonsumo simultáneo; edad promedio de inicio 16 años; combinaciones más comunes fueron alcohol + tabaco + marihuana y tabaco + alcohol + drogas de prescripción. Aprenden de las combinaciones de drogas a través de amigos, consumen en grupos mixtos, fuera de la universidad (clubes nocturnos) y lo hacen para relajarse o disfrutar de la compañía de amigos. Es fundamental desarrollar estrategias integrales si se desea contribuir a mejorar la salud de esta población de jóvenes, quienes estarán a cargo de la salud de las generaciones futuras.

DESCRIPTORES: Estudiantes. Drogas ilicitas. Conducta adictiva. Trastornos relacionados con substancias. Factores de riesgo.

\section{CHARACTERISTICS OF SIMULTANEOUS POLYDRUG USE AMONG UNDERGRADUATE STUDENTS OF HEALTH SCIENCES PROGRAMS OF ONE UNIVERSITY, SANTIAGO - CHILE}

\begin{abstract}
Cross sectional study whose objective was to establish the patterns of simultaneous poly-drug use among students in undergraduate medical and nursing careers, at a university in Santiago - Chile. A sample of 188 students was studied. Results: 42.3\% of students refered simultaneous poly-drug use, average age of onset of 16 years; most common combinations were alcohol + tobacco + marijuana and tobacco + alcohol with prescription drugs. They learn about drug combinations through friends, consume in mixed groups outside the university (nightclubs) and do it to relax or enjoy the company of friends. It is essential to develop comprehensive strategies in order to improve the health of this population of youngsters, who will be responsible for the health of future generations. DESCRIPTORS: Students. Street drugs. Behavior addictive. Substance-Related disorders. Risk factors.
\end{abstract}

\section{CARACTERISTICAS DO POLICONSUMO SIMULTÂNEO DE DROGAS EM ALUNOS DE GRADUAÇÃO DA ÁREA DE CIÊNCIAS DA SAÚDE EM UMA UNIVERSIDADE, SANTIAGO - CHILE}

RESUMO: Estudo transversal, cujo objetivo foi estabelecer os padrões de policonsumo de drogas entre estudantes de Medicina e de Enfermagem, em uma universidade de Santiago - Chile. A amostra foi composta de 188 alunos. Os resultados indicam que $42,3 \%$ dos alunos são poli-consumidores, simultaneamente, com idade média de aparecimento de 16 anos e as combinações mais comuns foram álcool + tabaco + maconha e tabaco + álcool + medicamentos prescritos, além de aprendizagem através de amigos, e consumido em grupos mistos fora da universidade (discotecas) para fazê-los relaxar ou aproveitar a companhia dos amigos. É essencial desenvolver estratégias globais, se quiser contribuir para melhorar a saúde da população, e particularmente a este grupo de jovens, que serão responsáveis pela saúde de gerações futuras.

DESCRITORES: Estudantes. Drogas ilicitas. Comportamento aditivo. Transtornos relacionados ao uso de substancias. Fatores de risco. 


\section{INTRODUCCIÓN}

Las tendencias mundiales han reflejado un aumento en el uso de drogas lícitas e ilícitas, en relación a la magnitud del consumo, a la morbimortalidad asociada a esta y al uso de sustancias entre los estudiantes universitarios, considerándose como un problema de salud pública en algunos países. ${ }^{1}$ A nivel regional, existe una creciente preocupación por los estudiantes universitarios de América Latina y el Caribe, y específicamente en relación al policonsumo de sustancias, debido a que este consumo pondría en peligro su bienestar, al favorecer la participación de éstos en comportamientos de riesgo, y aumentando la carga del cuidado en cada país.

El consumo de sustancias es más prevalente en la población entre 18 y 25 años, ${ }^{2}$ edad que coincide con la vida universitaria. Sin ir más lejos, en nuestra región, los jóvenes universitarios presentan la más alta prevalencia de consumo de drogas alguna vez en la vida y en el último año. ${ }^{3-4}$ Esta alta prevalencia sumado a la vulnerabilidad que caracteriza a este grupo, lo constituyen en la población objetivo de este estudio. ${ }^{5-6}$

En Chile es coincidente la situación a nivel regional: la población entre 18 y 25 años, tiene la más alta prevalencia de vida y prevalencia de consumo en el último año, en el uso de drogas lícitas e ilícitas, y se estima que cerca del 30\% de los jóvenes de esta edad, presenta un consumo de alcohol considerado como "problemático". ${ }^{4}$ La principal sustancia consumida es el alcohol. No obstante, se ha observado que el consumo de sustancias como marihuana y cocaína, se ha duplicado. ${ }^{7}$ El consumo elevado de sustancias ilícitas en estos jóvenes se relaciona con problemas en su círculo familiar, académico y laboral, así como en la sociedad en la que se ven inmerso. ${ }^{4}$

En relación a las carreras, el consumo tanto en cantidad como tipo de sustancia varía, siendo más alto en carreras del área de ingeniería, ciencias sociales y humanismo, intermedio para carreras de ciencias de la salud, y bajo en carreras de educación. ${ }^{8}$ Los estudiantes de las ciencias de la salud son de particular interés, al ser profesiones que están involucradas en el cuidado de la salud de la población, lo que puede ser más estresante y exigente que otras áreas de estudio.

En general, los datos disponibles sobre el uso indebido de drogas en estudiantes universitarios se centran en la utilización de una única sustancia (solo alcohol o solo marihuana), prestando poca atención al uso simultáneo de varias drogas. Esto se observa especialmente en países en vías de desarrollo, de regiones como el Caribe y América Latina.

Por otra parte, dichos estudios han concentrado los esfuerzos en carreras universitarias identificadas como vulnerables a este consumo, como son las carreras del área social y humanista, y escasean los estudios en estudiantes del área de la salud. Este grupo tiende a ser insuficientemente representado en los estudios de esta naturaleza, ya que a menudo no son considerados como usuarios de sustancias; ${ }^{9} \mathrm{y}$ sin embargo, es en ellos donde el consumo de sustancias lícitas e ilícitas, no es solo perjudicial para el propio individuo, sino también para quienes le rodean, al ser proveedores de servicios y tener bajo su responsabilidad el cuidado de la salud de la población.

Este estudio se centró principalmente en establecer los patrones de policonsumo simultáneo de drogas en estudiantes universitarios de pregrado de carreras de ciencias de la salud, en una universidad de la ciudad de Santiago - Chile, entendiendo por policonsumo simultáneo la coingestión de diferentes drogas al mismo tiempo ${ }_{1}^{10} \mathrm{y}$ fundamentado en el hecho de que el policonsumo simultáneo expone a los estudiantes universitarios a un mayor riesgo de consecuencias inmediatas y en el largo plazo, a un daño importante.

El estudio fue guiado bajo la Teoría Biopsicossocial, por su amplitud, integración y capacidad para concentrarse en las principales características del fenómeno de policonsumo. La teoría postula que el uso de sustancias es el resultado de una compleja interacción entre una combinación de aspectos biológicos, psicológicos, sociales, culturales, espirituales y factores determinantes. ${ }^{11}$

Lo objetivo fue establecer los patrones de policonsumo simultáneo de drogas en estudiantes universitarios de pregrado de carreras de medicina y enfermería, en una universidad de la ciudad de Santiago - Chile.

\section{METODOLOGÍA}

Estudio de tipo transversal que forma parte de un estudio multicéntrico. La población objetivo fue de estudiantes universitarios de pregrado de las carreras de Medicina y Enfermería, de primer y segundo año, mayores de 18 años, que estuvieran matriculados a tiempo completo o parcial, en una universidad de la ciudad de Santiago - Chile.

La muestra fue calculada del total de los alumnos de primer y segundo año de las carreras 
mencionadas (estimado en 433 estudiantes), usando el programa Sample Size Calculation Programme for Survey System. Este programa está basado en un intervalo de confianza de $95 \%$, calculándose sobre el predominio de tiempo de vida de empleo de alcohol en la población general del país, obteniéndose una muestra estimada en 188 estudiantes

Con el fin que todos los estudiantes tuvieran igual chance de participar, se seleccionaron al azar cuatro cursos de la lista total de cursos obligatorios de cada carrera y nivel participante, cuya suma total de estudiantes permitió cumplir con la muestra necesaria de 188 participantes. La participación fue voluntaria y bajo autorización dada en un documento de consentimiento informado.

Para la recolección de los datos se utilizó un cuestionario anónimo, auto-administrado, construido por los investigadores. En él se incorporaron además algunas preguntas de instrumentos publicados en la literatura, con la respectiva autorización de los autores, tales como: "Reasons for drug", 12 "Wasting the best and the brightest: Substance abuse at America's colleges and universities", Canadian Survey, ${ }^{13}$ CAGE. ${ }^{14}$ El cuestionario quedó conformado por cuatro secciones y un total de 58 preguntas cerradas, destinadas a indagar sobre las características socio-demográficas y de uso de sustancias psicoactivas. El instrumento fue elaborado en idioma inglés, traducido al español y contra-traducido a su idioma original para incrementar su validez idiomática. Adicionalmente, este fue sometido a una prueba piloto y análisis factorial, realizándose los ajustes necesarios previos a la aplicación.

El reclutamiento de los estudiantes fue realizado por el investigador principal, posterior a la autorización por parte de las autoridades de la universidad. La recolección de datos se realizó en las salas de clases, en ausencia del profesor a cargo del curso, y bajo la conducción del investigador principal y un ayudante capacitado. En primera instancia se les explicó a los estudiantes los objetivos y características generales del proyecto, que incluyó la lectura en voz alta por parte del investigador del consentimiento informado y del cuestionario, posterior a los cual se dio tiempo para preguntas. Una vez firmado el consentimiento y llenado el cuestionario, los estudiantes depositaron los documentos en dos cajas selladas (separadas), manteniendo así la seguridad y confidencialidad de los datos recolectados.

Los datos fueron analizados descriptivamente, determinándose la distribución de frecuencias y las relaciones lógicas que se pudieran dar entre las variables en estudio. Las relaciones entre variables categóricas fueron analizadas mediante la prueba de Chi-Cuadrado, y a aquellas entre variables continuas se les aplicaron pruebas de correlación de Pearson. Se consideró un nivel de confianza de 95\%. Para el análisis de los datos, se utilizó el programa estadístico SPSS versión 15 para Windows.

La investigación contó con la aprobación del Research Ethics Board (REB) del Centre for Addiction and Mental Health (CAMH) (Protocol reference \#225/2008) y los Comités de Ética de la Escuela de Enfermería (DIR 03/09) y Comité de Ética de la Escuela de Medicina (C.E \# 79/ 09), de la Pontificia Universidad Católica de Chile.

\section{RESULTADOS}

\section{Características sociodemográficas de la muestra}

Un $73.9 \%$ fue de sexo femenino y las edades se concentraron en el rango entre 18 y 20 años $68.09 \%$. Un $96.3 \%$ refirió ser soltero(a), $77.1 \%$ vivir en casa con sus familias, y un $81.9 \%$ consideró como algo muy importante o importante la religión o creencia. Un $77.1 \%$ de los estudiantes pertenecía a la carrera de Enfermería y un $67.6 \%$ cursaba segundo año de su carrera al momento de la recolección de los datos.

\section{Características generales de consumo}

El 58.5\% de los estudiantes manifestó haber consumido sustancias psicoactivas al menos una vez en la vida, siendo los 15 años la edad promedio de inicio del consumo. El 51.1\% había consumido en los últimos 12 meses, principalmente sustancias como alcohol $92.7 \%$, tabaco $43.8 \%$ y marihuana $24 \%$, escogiendo los fines de semana para realizar el consumo. Con respecto a la carrera de procedencia del estudiante, mientras que en medicina el $72.1 \%$ de los jóvenes sí ha consumido alguna vez en su vida una sustancia psicoactiva, en el caso de enfermería esta proporción decrece al $54.5 \%$; diferencia que resultó ser significativa $(p=0,04)$.

El 59.3\% de los jóvenes que consideran muy importante su religión no han consumido droga en los últimos 12 meses. Esta cifra decrece a $44.4 \%$ en los jóvenes para los que la religión es importante, a $29.4 \%$ para quienes la religión es "algo importante", y a $11.8 \%$ para quienes no es importante. 


\section{Características del policonsumo simultáneo}

Un 42.3\% (n=41) refirió haber utilizado más de una sustancia psicoactiva al mismo tiempo en los últimos 12 meses, mientras que en los últimos 30 días esta cifra baja a un $30.9 \%$. La edad promedio del inicio de policonsumo fue de 16 años.

La tabla 1 muestra las principales combinaciones de sustancias según su consumo en los últimos 12 meses y 30 días. En ella destaca que la combinación más frecuente es alcohol + tabaco + marihuana, con una frecuencia de 10 casos, para los últimos 12 meses. Para esta misma combinación, la cifra baja a dos casos en los últimos 30 días. En segundo lugar aparece la combinación de drogas de prescripción + tabaco o alcohol, con cuatro casos cada combinación en los últimos 12 meses y 30 días.

Tabla 1 - Frecuencia de policonsumo simultáneo de sustancias psicoactivas durante los últimos 12 meses y 30 días, según tipo de sustancia, Santiago-Chile, 2009

\begin{tabular}{lcc}
\hline Tipo de sustancia & $\begin{array}{c}\text { últimos } \\
\text { 12 meses }\end{array}$ & $\begin{array}{c}\text { últimos } \\
\mathbf{3 0} \text { días }\end{array}$ \\
\hline Alcohol + marihuana & 9 & 3 \\
Alcohol + cocaína & - & - \\
Alcohol + drogas prescritas & 4 & 4 \\
Alcohol + tabaco + marihuana & 10 & 2 \\
Alcohol + cocaína + tabaco & - & - \\
Alcohol + cocaína + marihuana & - & - \\
+ tabaco & 8 & 2 \\
Tabaco + marihuana & 4 & 4 \\
Tabaco + drogas prescritas & - & - \\
Marihuana + cocaína & 1 & 1 \\
Marihuana + drogas prescritas & & \\
\hline
\end{tabular}

En relación a la forma en que los estudiantes aprendieron acerca de la combinación de sustancias psicoactivas, la forma más usada es el aprendizaje a través de un amigo con un $14.4 \%$, seguido por "por mi mismo" $13.4 \%$. Los lugares donde realizan este tipo de consumo son "fuera de la universidad/en clubes nocturnos" $33.8 \%$, en fiestas en la universidad $23.9 \%$ y otros sitios, como la casa de amigos $18.3 \%$. Las sustancias fueron adquiridas tanto fuera como dentro de la misma universidad $28.7 \%$ y $16.5 \%$ respectivamente.

Sobre un total de 28 casos considerados válidos para estas preguntas, se observó que los grupos donde los estudiantes consumen son mayoritariamente mixtos $75.0 \%$; consiguen las sustancias por ellos mismos, y las adquieren ya sea con dinero propio $60.7 \%$ o solicitan dinero a un miembro de la familia $25.0 \%$. La decisión del tipo de sustancia y la combinación de éstas para el consumo en forma simultánea fue referida como individual $64.3 \%$.

Los estudiantes perciben como fácil o muy fácil $72.3 \%$ el acceso a las sustancias psicoactivas. Resulta importante destacar que un $27.1 \%$ manifestó no tener conocimiento respecto a cómo adquirirlas. Al consultarles en relación a las drogas de prescripción y como logran acceder a ellas, la opción más seleccionada fue "conseguir este tipo de sustancias a través de amigos", seguido por la opción de "conseguirlas a través de un proveedor".

Respecto a la manera en que los estudiantes perciben el consumo de sustancias psicoactivas en la universidad, un $22 \%$ respondió que es un problema muy importante, un $20 \%$ que es un problema poco importante, un $22 \%$ no lo considera un problema, y un 35\% manifestó no saber. En relación a la percepción de los jóvenes sobre el consumo de sustancias psicoactivas entre los profesionales del área de la salud, un 39.9\% de los jóvenes consideró que es un gran problema, un $19.5 \%$ que es un problema menor y un $6.9 \%$ que no es un problema.

Tabla 2 muestra las razones seleccionadas por los estudiantes para el policonsumo simultaneo de sustancias psicoactivas. En cuanto a las categorías agrupadas, los cambio de ánimo y propósitos sociales fueron los aspectos referidos con mayor frecuencia. Por su parte, las razones específicas más referidas para el policonsumo simultáneo fueron: "le ayuda a relajarse" (22 casos), "le ayuda a disfrutar la compañía de sus amigos" (18 casos)", le ayuda a dejar de preocuparse por un problema" (12 casos), y "le ayuda a sentir emoción o euforia" (12 casos). 
Tabla 2 - Razones seleccionadas por los estudiantes para consumir más de una sustancia psicoactivas en forma simultánea, Santiago-Chile, 2009

\begin{tabular}{|c|c|c|c|}
\hline Aspecto central & Razones & Frecuencias & $\begin{array}{l}\text { Promedio de } \\
\text { frecuencias }\end{array}$ \\
\hline \multirow{5}{*}{ Cambio de ánimo } & Le hace sentirse mejor cuando está deprimido & 5 & \multirow{5}{*}{10,6} \\
\hline & Le ayuda a dejar de preocuparse por un problema & 12 & \\
\hline & Le ayuda a relajarse & 22 & \\
\hline & Le ayuda a sentir emoción o euforia & 12 & \\
\hline & Para sentirse fuertemente drogado o intoxicado & 2 & \\
\hline \multirow{4}{*}{ Efectos físicos } & Mejora las sensaciones durante las relaciones sexuales & 5 & \multirow{4}{*}{4,25} \\
\hline & Le ayuda a permanecer despierto & 7 & \\
\hline & Le ayuda a perder peso & 1 & \\
\hline & Le ayuda a dormir & 4 & \\
\hline \multirow{5}{*}{ Propósitos sociales } & Le ayuda a disfrutar la compañía de sus amigos & 18 & \multirow{5}{*}{10} \\
\hline & Le ayuda a sentir más confianza & 8 & \\
\hline & Le ayuda a desinhibirse & 11 & \\
\hline & Le ayuda a mantenerse bien cuando sale con sus amigos & 10 & \\
\hline & Para relacionarse mejor con los demás & 3 & \\
\hline \multirow{3}{*}{$\begin{array}{l}\text { Facilitación de } \\
\text { actividades }\end{array}$} & Le ayuda a concentrarse en trabajo o estudio & 4 & \multirow{3}{*}{4,67} \\
\hline & $\begin{array}{l}\text { Incrementa las actividades como jugar deporte, escuchar } \\
\text { música }\end{array}$ & 4 & \\
\hline & Le ayuda a hacer algo cuando está aburrido & 6 & \\
\hline \multirow{2}{*}{$\begin{array}{l}\text { Manejo de los efectos } \\
\text { de otras sustancias }\end{array}$} & Mejora los efectos de otras sustancias & 4 & \multirow[b]{2}{*}{2} \\
\hline & $\begin{array}{l}\text { Le ayuda a atenuar los efectos posteriores de otras } \\
\text { sustancias }\end{array}$ & - & \\
\hline
\end{tabular}

Finalmente, consideramos importante hacer notar que tres estudiantes que refirieron poli consumo simultáneo manifestaron haberse sentido presionados para iniciar este tipo de consumo $7.3 \%$, mientras que siete refirieron haber influenciado a otros $17.1 \%$.

\section{DISCUSIÓN}

El uso de sustancias psicoactivas en la población joven se ha constituido en los últimos años en una problemática a nivel mundial, aspecto que radica en el hecho de que estos jóvenes constituyen lo que conocemos como adultos emergentes, es decir, individuos aun en formación pero con responsabilidades presentes y futuras.

Esta investigación registró en una primera instancia las características generales del consumo de sustancias psicoactivas en estudiantes de nivel universitario de primer y segundo año de dos carreras de ciencias de la salud (medicina y enfermería), para posteriormente concentrarse en los aspectos centrales del poli consumo y sus características.

En términos de edad, los jóvenes se concentraron entre 18 y 20 años, intervalo que coincide con la edad de mayor prevalencia de consumo de drogas en Chile, en población joven. ${ }^{15}$
El alcohol fue la sustancia psicoactiva más mencionada para el consumo de los últimos 12 meses y 30 días, tal como se aprecia en estudios realizados en Norteamérica, ${ }^{10}$ seguido por el consumo de tabaco, marihuana y drogas de prescripción. A diferencia de los estudios analizados sobre la situación Chilena de la última década, ${ }^{15}$ el porcentaje de estudiantes que refirió consumir marihuana en nuestra muestra fue considerablemente menor, comparado con alcohol y tabaco. Esto podría deberse a la alta representación femenina en nuestra muestra, y a factores sociales y educacionales específicos de nuestra población estudiada, comparados con la realidad nacional.

Consideramos preocupante que la mayoría de las mujeres en la muestra refirió haber consumido sustancias psicoactivas al menos una vez en su vida. Esto podría ser concordante con reportes a nivel mundial, en los que la prevalencia de consumo en mujeres ha aumentando para distintas sustancias psicoactivas en relación a la de los hombres. ${ }^{15}$

Con respecto al policonsumo, la literatura registra la asociación mayoritariamente de alcohol y tabaco como drogas principales, combinadas con otras sustancias, ${ }^{10,16-17}$ lo que coincide con lo encontrado en este estudio. 
La edad de inicio promedio referida en nuestra muestra fue de 15 años para el consumo general y 16 años para el policonsumo, cifras similar a la comúnmente reportadas en la literatura, donde se señala que los jóvenes en general inician este tipo de consumo a edad escolar ${ }^{10,16,18}$. Frente a este aspecto, sería adecuado considerar la realización de futuras investigaciones en estudiantes de educación secundaria, de manera de poder obtener una visión amplia en relación al ciclo vital y cómo los distintos cambios que van sufriendo en su proceso de maduración influye en la conductas de policonsumo.

La religión aparece como un elemento asociado al menor consumo, ya que los estudiantes que consideran a la religión o creencia como importante o muy importante, mostraron menores niveles de uso de drogas psicoactivas.

En cuanto a las razones del consumo, las situaciones vividas y las sensaciones que experimentan al combinar distintas sustancias son las más seleccionadas. Entre ellas, la necesidad de relajarse, de disfrutar de la compañía de amigos, de experimentar euforia o "nuevas sensaciones", y la posibilidad de evadir por un breve instante aquellas situaciones que les producen tristeza o preocupación, son las más ampliamente referidas por los jóvenes de este estudio, y que coinciden en general con lo expuesto en la literatura. ${ }^{18}$

Los factores que presentan relación con el policonsumo que emergieron en esta investigación, son coherentes con lo propuestos en el modelo planteado, en base a la teoría biosicosocial, donde se espera que aspectos como la creencia, el estrés y la relación con los pares y familias, influencien la conducta de consumo y determinen características del patrón de policonsumo.

Sin lugar a dudas que el consumo de sustancias psicoactivas es un problema multifactorial, que conlleva a múltiples consecuencias tanto para el estudiante como para su entorno. Nos parece preocupante que futuros profesionales, enfermeras y médicos, sean consumidores de sustancias psicoactivas, dado las potenciales consecuencias que a corto y largo plazo esto puede traer tanto para sus vidas como para su desempeño profesional. Por estas razones, consideramos fundamental mejorar los programas de prevención existentes, y construir nuevas estrategias que permitan aminorar esta problemática. El desarrollo de iniciativas integrales, tanto a nivel local como regional, son acciones prioritarias si se desea contribuir a mejorar la salud de la población, y en particular de este grupo de jóvenes, que estarán a cargo de la salud de las generaciones futuras y que deberán velar por un mejor futuro en este ámbito.

\section{CONCLUSIONES}

Este estudio permitió explorar las características del policonsumo simultáneo de sustancias psicoactivas en estudiantes universitarios de pregrado de las carreras de enfermería y medicina. Las principales combinaciones se realizan con tres tipos de sustancias, tabaco, alcohol y marihuana, destacando el inicio temprano. Este elemento es fundamental a la hora de desarrollar políticas de prevención en salud, tanto a nivel escolar como universitario, concentrando los esfuerzos en edades donde los jóvenes son vulnerables.

\section{Limitaciones}

Se observó desmotivación por parte de los estudiantes a participar de este estudio, debido a que los meses de recolección de datos coincidieron con la aplicación de encuestas o cuestionarios de otros proyectos de la universidad participante. Por otra parte, la participación del estudiante con respecto al desarrollo del cuestionario fue disímil. Varios de los estudiantes que refirieron policonsumo omitieron preguntas centrales en torno al tema, lo que redujo la muestra, limitando las conclusiones. Esto además puede haber generado diferencias sistemáticas que distorsionen la validez de los resultados.

Los resultados obtenidos en este estudio no son generalizables a la población de jóvenes en general o a la de estudiantes universitarios, ya que sólo se incluyeron estudiantes de primero y segundo años de las carreras de Medicina y Enfermería en una universidad en Chile.

\section{Recomendaciones}

Debido a que la evidencia apunta a que los estudiantes inician tempranamente el policonsumo simultáneo de sustancias psicoactivas, prácticamente tres o cuatro años antes de ingresar a la universidad, consideramos necesaria la realización de futuras investigaciones en estudiantes de educación secundaria, de tal forma de poder obtener una visión amplia en relación al ciclo vital, y de cómo los distintos cambios que van sufriendo en su proceso de maduración influye en la conducta de policonsumo. 


\section{AGRADECIMIENTOS}

Los autores agradecen al gobierno de Canadá/DFAIT, a la Organización de los Estados Americanos (OEA), Comisión Interamericana para el Controle del Abuso de Drogas (CICAD), al Centre for Addiction and Mental Health (CAMHToronto/Canadá), a los estudiantes y autoridades de la universidades participante del estudio y demás colaboradores. Un agradecimiento especial al Dr. Gustavo Mery, CAMH Reviewer, por su colaboración en la revisión y editoración final de todos los trabajos y informes del grupo III.

\section{REFERENCIAS}

1. World Health Organization. Global status report on alcohol [monograph on the internet]. Washington: WHO; 2004 [cited 2007 Nov 20]. Available from: http//www.who.int/substance_abuse/ publication/global_status_report_2004_overview. pdf

2. World Health Organization. Neuroscience of psychoactive substance use and dependence. Summary [monograph on the internet]. Washington: WHO; 2004 [cited 2008 Jul 26]. Available from: http// www.who.int/substance_abuse/publications/en/ neuroscience.pdf

3. Carlini E, Galduroz J, Noto A, Nappo A. II Levantamento domiciliary nacional sobre o uso de drogas psicotrópicas: estudo envolvendo as 108 maiores cidades do país.- National Institute of drugs abuse [cited $2008 \mathrm{Jul}$. Available from: http:/ / www. unodc.org/pdf/brazil//II\%20Levantamento \% 20 Domiciliar\%20Dr\%20Elisaldo\%Carlini_alterado2. pdf.

4. Consejo Nacional para el Control de Estupefacientes. Séptimo estudio nacional de drogas en población general de Chile, 2007.Informe de resultados generales. Santiago: Consejo Nacional para el Control de Estupefacientes; 2007.

5. Laranjo $\mathrm{T}$, Soares $\mathrm{C}$. University residence halls: socialization processes and drug consumption. Rev Saude Pública [online]. 2006 [acceso 2008 Jul 17]; 40(6):1-7. Disponible en: http://www.scielo.br/ $\mathrm{pdf} / \mathrm{rsp} / \mathrm{v} 40 \mathrm{n} 6 / \mathrm{en} \_10 . p d f$

6. Simons J, Gaher R, Correia C, Hansen C, Christopher $\mathrm{M}$. Anaffective-motivational model of marijuana and alcohol problems among college students. Psychol Addict Behav [online]. 2005 [acceso 2008 Aug 8]; 19(3):326-34. Disponible en: http:/ / psycnet.apa.org/ index.cfm?fa=buy.optionToBuy\&id=2005-11382-013
7. National Centre on Addiction and Substance Abuse. Wasting the best and the brightest: Substance abuse at America's colleges and universities [CASA]. Columbia University; 2007.

8. Gómez A, Herde J, Laffee A, Lobo S, Martín E. Consumo de drogas lícitas e ilícitas por estudiantes universitarios. Rev Fac Ciencias Salud Universidad de Carabobo [online]. 2007 [acceso 2008 Jul 17]; 11(3):41-5: Disponible en: http://bases.bireme.br/ cgi-bin/wxislind.exe/iah/online/?IsisScript=iah/ iah. $x$ is\&src $=$ google\&base $=$ ADOLEC\&lang $=p \& n e x$ tAction $=$ lnk\&exprSearch $=502852$ \&indexSearch $=I D$

9. Maddux J, Hoppe S, Costello R. Psychoactive substance use among medical students. Am J Psychiatry [online]. 1986 [acceso 2008 Jul 4]:143(2):18791. Disponible en: http:/ / ajp.psychiatryonline.org/ cgi/content/abstract/143/2/187

10. McCabe SE, Cranford JA, Morales M, Young A. Simultaneous and concurrent poly-drug use of alcohol and prescription drugs: prevalence, correlates, and consequences. J Stud Alcohol. 2006 Jul; 67(4):529-37.

11. Huyse F, Lyons J, Stiefel F, Slates J, Jonge P, Latour C. Operationalizing the biopsychosocial model: the Intermed. Psychosomatics. 2001 Jan-Feb; 42(1):5-13.

12. Boys A, Marsden J, Strang J. Understanding reason for drug use among young people: a functional perspective. Health Educ Res. 2001 Aug; 16(4):45769.

13. Centre of Addiction and Mental Health and Université de Montreal. Canadian Campus Survey 2008. Toronto, Canadá.

14. Erwing J. Detecting alcoholism. The CAGE questionnaire. JAMA 1984; 252(14):1905-7.

15. Consejo Nacional para el Control de Estupefacientes. Sexto estudio nacional de drogas en población general de Chile, 2004. Consumo de drogas en jóvenes. Santiago $(\mathrm{CH})$ : Consejo Nacional para el Control de Estupefacientes; 2007.

16. O'Reily BO, Jessen J. Cannabis and other drug use by tertiary students in Darwin, Northern Territory, Australia. South Pacific. J Psycho [online]. 2005 [acceso 2008 Out 1]; 16(1): Disponible en: http:// spjp.massey.ac.nz/issues/2005-v16/v16-oreilly.pdf

17. Barrett S, Darredeau C, Pihl R. Patterns of simultaneous poly-substance use in drug using university students. Hum Psychopharmacol. 2006 Jun; 21(4):255-63.

18. Webb E, Ashton CH, Kelly P, Kamali F. Alcohol and drug use in UK university student. Lancet. 1996 Oct 5; 348(9032):922-5. 